Check for updates

Cite this: RSC Adv., 2017, 7, 34415

Received 21st February 2017

Accepted 23rd June 2017

DOI: $10.1039 / \mathrm{c} 7 \mathrm{ra02146a}$

rsc.li/rsc-advances

\section{Enhancing the proliferation of MC3T3-E1 cells on casein phosphopeptide-biofunctionalized 3D reduced-graphene oxide/polypyrrole scaffolds $\uparrow$}

\author{
Weibo Jie, $t^{\text {ad }}$ Fuxiang Song, (D) $t^{\text {bc }}$ Xiaocheng Li, ${ }^{c}$ Wen Li, ${ }^{b}$ Rui Wang, ${ }^{b}$ Yanjiao Jiang, ${ }^{b}$
} Libo Zhao, ${ }^{\mathrm{b}}$ Zengjie Fan, ${ }^{\mathrm{b}}$ Jizeng Wang ${ }^{\star a}$ and Bin Liu ${ }^{\star b}$

In bone tissue engineering, the 3D macro-scaffold plays an indispensable role as a matrix for cell proliferation, differentiation and tissue formation, for which a bioactive surface is required. Previously, we successfully prepared the 3D macro-reduced graphene oxide/polypyrrole (3D rGO/PPY) scaffold; in the present study, by using casein phosphopeptide (CPP) as a bioactive molecule, we have developed a 3D rGO/PPY/CPP composite scaffold through a simple, but low-cost electrostatic self-assembly method and have explored the application of the composite scaffold in bone tissue engineering. The results have indicated that the CPP successfully modified the backbone of the scaffold, and demonstrated that the developed 3D rGO/PPY/CPP scaffold has excellent hydrophilic behavior and water uptake performance, much better than that of 3D rGO/PPY. In the biomimetic mineralization experiment, the CPP-modified matrix, particularly 3D rGO/PPY/CPP20, could promote the rapid formation of hydroxyapatite in simulated body fluid solution on just the $1^{\text {st }}$ soaking day. On co-culturing with the MC3T3-E1 cells, the 3D rGO/PPY/CPP20 kept the cells at a higher proliferation state of 5.07 times that of the control group, superior to that of the 3D rGO/PPY/CPP10 (3.88 times) and the 3D rGO/PPY group (2.07 times). The excellent osteoblastic performance of the 3D rGO/PPY/CPP20 composite scaffold can be attributed to the good biological properties of CPP, and the unique 3D macro-structure with a high specific surface area. Our findings suggest that the 3D rGO/PPY/CPP20 can be considered as an attractive scaffold for bone healing and regeneration in the future.

\section{Introduction}

Bone tissue engineering is one of the most promising techniques in healing the bone defects caused by fracture, cancer resection, serious infection or osteo-degenerative diseases. ${ }^{\mathbf{1}}$ The concepts based on this technique can effectively avoid problems of donor site scarcity, immune rejection and pathogen transfer

${ }^{a}$ Key Laboratory of Mechanics on Disaster and Environment in Western China, The Ministry of Education, College of Civil Engineering and Mechanics, Lanzhou University, Lanzhou 730000, China.E-mail: jzwang@lzu.edu.cn

${ }^{b}$ School of Stomatology of Lanzhou University, Lanzhou 730000, China. E-mail: liubkq@lzu.edu.cn

'Laboratory of Clean Energy Chemistry and Materials, Lanzhou Institute of Chemical Physics, Chinese Academy of Sciences, Lanzhou, 730000, China. E-mail: xiaocheng@ licp.cas.cn

${ }^{d}$ Lanzhou University Second Hospital, Lanzhou 730000, China. E-mail: jiewb@lzu. edu.cn

$\dagger$ Electronic supplementary information (ESI) available: Fig. S1 low-magnification SEM images of 3D rGO/PPY (the first row), 3D rGO/PPY/CPP10 (the second row), and 3D rGO/PPY/CPP20 (the third row) composite scaffolds after being soaked in $1.5 \times \mathrm{SBF}$ at $37{ }^{\circ} \mathrm{C}$ for $1,3,5$, and 7 days, respectively. Fig. S2 growth mechanism of HA on backbone of the CPP-modified 3D rGO/PPY scaffold. See DOI: $10.1039 / \mathrm{c} 7 \mathrm{ra02146a}$

\$ These authors contributed equally to this work. that are encountered in autografting, allografting and xenografting. ${ }^{1,2}$ In bone tissue engineering, scaffolds play the indispensable roles of supporters for cell proliferation, differentiation and tissue formation. Also, their architecture roughly defines the ultimate configuration of the new bone and cartilage. $^{3}$ An ideal scaffold should have the following typical features: ${ }^{4}$ (1) three-dimensional cross-linked backbone with porous network for cell growth, nutrient and metabolic waste transportation; (2) proper mechanical properties to match those of the tissue at the defect site; ${ }^{8}$ (3) desirable surface chemical state for cell adhesion, proliferation, and differentiation. With these guidelines in mind, in a few recent studies, several scaffolds have been successfully developed, such as polyurethane, (PU), ${ }^{5}$ biphasic calcium phosphate (BCP), ${ }^{6}$ polyethylene glycol (PELA), ${ }^{7}$ and $3 \mathrm{D} \mathrm{rGO} / \mathrm{PPY}^{8}$ On the whole, these scaffolds can function as alternative 3D matrixes for bone tissue engineering. However, these scaffolds are usually hydrophobic and lack bioactivity, preventing cell adhesion and proliferation. ${ }^{5-9}$ Some reports also claim that the implant materials without bioactivity cannot ultimately form a good osseointegration with new bone, and angular absorption, prolonging the healing time and even implant failure are generally involved. ${ }^{\mathbf{1 0 , 1 1}}$ Therefore, plenty of experimental methods, such as plasma treatment, ozone or 
photo-induced grafting, and surface oxidation strategies are generally needed to introduce some hydrophilic/bioactivity components onto the surfaces of the matrix. ${ }^{12,13}$ Unfortunately, special/expensive equipment and complex-stepped fabrication processes are generally involved, thus limiting the applications in bone tissue engineering. It is therefore essential to develop a simple, but low-cost method for functionalizing the surface of the 3D rGO/PPY composite scaffold and endowing the scaffold surface with good biocompatibility, if possible, to mediate or boost the nucleation and growth of hydroxyapatite in vivo.

Casein phosphopeptides (CPP) are natural bioactive peptides obtained from bovine casein via biotechnology. They possess good biological activity and are capable of promoting the absorption of calcium ions in vivo because of the numerous phosphate groups in CPP molecules that create polar and acidic domains and are thus more favourable for bonding $\mathrm{Ca}^{2+}$ ions, even in neutral and alkaline surrounding. ${ }^{\mathbf{1 4}}$ Besides, the CPP molecules contain several negatively charged amino acid sequences, which consist of glutamic acid and phosphorylated serine residues (Ser[P]-Ser[P]-Ser[P]-Glu-Glu). ${ }^{15}$ Also, these short amino acid sequences on CPP molecules were proved to be the molecular determinants for their biological activities on osteoblast-like cells. ${ }^{16}$ Tsuchita and co-workers reported that CPP could improve the solubility of calcium and increase the calcification of embryonic rat bones in the diaphyseal area. ${ }^{17}$ Kantlehner et al. prepared peptide-coated polymethyl methacrylate (PMMA) pellets and implanted them into the patella groove of rabbits, indicating that they could be integrated into regenerating bone tissue faster and more strongly than uncoated pellets. ${ }^{13}$ Therefore, if we can graft the CPP molecules on the desirable 3D scaffolds, the cell proliferation and bone regeneration can be realized in vivo at the healing site.

The electrostatic self-assembly technique is a simple, inexpensive and powerful approach to modifying the surfaces of scaffolds in any shape. This method mainly involves the sequential adsorption of oppositely charged polyelectrolytes to form multilayer film-modified structures. The reaction process is mainly attributed to different electrostatic forces, including hydrogen bonding, hydrophobic interactions and covalent bonding. ${ }^{18}$ To date, the self-assembly technique has been widely used to introduce bio-macromolecules onto the surfaces of scaffolds in bone tissue engineering. Based on this facile strategy, we have successfully fabricated the 3D rGO/PPY scaffold, which has promising applications in bone tissue engineering. ${ }^{8}$ The adopted polymer molecules can arbitrarily alter the physiochemical properties of the 3D scaffold and endow the surface with positively or negatively charged states, which pave the way for assembling the CPP molecules on 3D rGO/PPY scaffolds and exploring their application in bone tissue engineering.

In the present study, we have successfully developed the PDDA functionalized-3D rGO/PPY scaffold by adsorbing the positively charged PDDA molecules on the 3D rGO/PPY scaffold to endow the scaffold surface with positive charges ${ }^{19}$ and then grafted the CPP molecules via an electrostatic self-assembly strategy. Results suggest that after grafting the CPP molecules, the developed 3D rGO/PPY/CPP scaffold demonstrates excellent hydrophilic behavior and water uptake performance, much better than that of the 3D rGO/PPY. Moreover, the 3D rGO/PPY/ CPP scaffold can effectively boost the mineralization of hydroxyapatite crystals in $1.5 \times$ Simulated Body Fluid (SBF) solution and the proliferation/differentiation of MC3T3-E1 cells, which is greatly superior to that of the $3 \mathrm{D}$ rGO/PPY scaffold, suggesting the intriguing application of the 3D rGO/PPY/ CPP scaffold in bone tissue engineering.

\section{Experimental section}

\subsection{Materials}

All of the reagents used in the experiment were analytical grade. Casein peptide phosphates (CPP) were purchased from Shanghai Source Biological Technology Co. Ltd. 1-Ethyl-3-(3dimethyl amino propyl) carbonyl imine (EDC), $N$-hydroxyl succinimide (NHS) and 2-(morphine) ethyl sulfonic acid (MES) were purchased from Aladdin Chemistry Co. Ltd. Poly (diallyl dimethylammonium) chloride (PDDA, 20 wt\%, Mw 100000200000) was purchased from Aladdin Chemistry Co. Ltd. The reagents AO, and EB were purchased from Sigma (St. Louis, MO, USA). BCIP/NBT Alkaline Phosphatase Colour Development Kit (no. C3206) was purchased from Sigma.

\subsection{Preparation of the 3D $\mathrm{rGO} / \mathrm{PPY} / \mathrm{CPP}$ composite scaffold}

The 3D rGO/PPY scaffold was prepared through an electrostatic LBL assembly strategy, followed by an electrochemical deposition process, as described in our previous report. ${ }^{8}$ Surface modification of 3D rGO/PPY with CPP molecules was also completed via an electrostatic self-assembly process and is described as follows. Firstly, the $3 \mathrm{D}$ rGO/PPY scaffold $(20 \times 20$ $\times 1.6 \mathrm{~mm}$ ) was subjected to an oxygen plasma treatment process to increase the water wettability of the sample and introduce negatively charged groups (such as hydroxyl and carboxyl groups) onto the scaffold surface, then soaked in 0.5 $w t \%$ PDDA solution for $2 \mathrm{~h}$ to reverse the charge state of the scaffold surface. Secondly, $10 \mathrm{~g} \mathrm{~L}^{-1}$ and/or $20 \mathrm{~g} \mathrm{~L}^{-1}$ of CPP solution were added to the mixed solution consisting of EDC $\left(20 \mathrm{mmol} \mathrm{L}^{-1}\right)$, NHS $\left(50 \mathrm{mmol} \mathrm{L}^{-1}\right)$ and $\operatorname{MES}\left(100 \mathrm{mmol} \mathrm{L}^{-1}\right)$. Thirdly, PDDA-anchored 3D rGO/PPY scaffolds were immersed into the abovementioned solution and allowed to stand overnight at $4{ }^{\circ} \mathrm{C}$ to assemble the CPP molecules on the positively charged scaffold through the electrostatic adsorption process. The 3D rGO/PPY scaffolds decorated by $10 \mathrm{~g} \mathrm{~L}^{-1}$ and/or $20 \mathrm{~g} \mathrm{~L}^{-1}$ CPP solution were finally denoted as 3D rGO/PPY/CPP10 and 3D rGO/PPY/CPP20, respectively.

\subsection{Characterization}

The surface morphology, crystalline structure and physicochemical state of the prepared samples were characterized by scanning electron microscope (SEM, JSM-5601LV) equipped with an energy dispersive X-ray analyzer (EDX), X-ray photoelectron spectroscopy (XPS, Kratos AXIS Ultra DLD, Al K $\alpha$ ), X-ray powder diffractometer (XRD, D/max-2400, Rigaku, $\mathrm{Cu} \mathrm{K \alpha}$ ) and Fourier infrared spectrometer (FT-IR, IFS66V/S, Bruker). 


\subsection{Contact angle and water uptake measurement}

Water contact angle (WCA) of the 3D rGO/PPY based composite scaffold was tested on a tensiometer (DSA100, KRUSS Co. Ltd., Germany) equipped with image analysis software using the sessile drop method. Typically, deionized water droplets with volume of $8 \mu \mathrm{L}$ were gently positioned on the materials through a micro-syringe and the images were captured to measure the angle formed at the liquid-solid interface. The contact angle value was calculated based on six individual measurements. Water uptake and retention rates of 3D rGO/PPY based scaffold were evaluated using an established method in literature. ${ }^{20}$ In detail, the dry scaffolds were weighed $\left(W_{\mathrm{O}}\right)$ prior to absorbing the water, then were immersed in distilled water overnight, dipped residual water with filter paper, and weighed $\left(W_{1}\right)$ to determine the water uptake rate. This was followed by drying the samples by centrifugation, the weighing again $\left(W_{\mathrm{D}}\right)$ to get the water retention rates. The percent equilibria of the water uptake rate $\left(W_{\mathrm{U}}\right)$ and water retention rate $\left(W_{\mathrm{R}}\right)$ were calculated using eqn (1) and (2)

$$
\begin{aligned}
& W_{\mathrm{U}}=\left[\left(W_{1}-W_{\mathrm{O}}\right) / W_{\mathrm{O}}\right] \times 100 \% \\
& W_{\mathrm{R}}=\left[\left(W_{\mathrm{D}}-W_{\mathrm{O}}\right) / W_{\mathrm{O}}\right] \times 100 \%
\end{aligned}
$$

\subsection{Biomimetic mineralization}

The bone-forming activities of the prepared 3D rGO/PPY, 3D $\mathrm{rGO} / \mathrm{PPY} / \mathrm{CPP} 10$ and 3D rGO/PPY/CPP20 scaffolds in vitro were evaluated in $1.5 \times \mathrm{SBF}$, which is composed of $\mathrm{Na}^{+}(213.0 \mathrm{mM})$, $\mathrm{K}^{+}(7.5 \mathrm{mM}), \mathrm{Mg}^{2+}(2.25 \mathrm{mM}), \mathrm{Ca}^{2+}(3.75 \mathrm{mM}), \mathrm{Cl}^{-}(22.17 \mathrm{mM})$, $\mathrm{HCO}_{3}{ }^{-}(6.3 \mathrm{mM}), \mathrm{HPO}_{4}{ }^{2-}(1.5 \mathrm{mM}), \mathrm{SO}_{4}{ }^{2-}(0.75 \mathrm{mM}) .{ }^{14}$ All scaffolds were soaked in $1.5 \times \mathrm{SBF}$ for different periods $(1,3,5$, and 7 days, respectively) in a thermostatic bath at $37^{\circ} \mathrm{C}$, followed by washing with copious amounts of water and finally dried at room temperature for further analysis.

\subsection{MTT assay}

The prepared 3D rGO/PPY, 3D rGO/PPY/CPP10, and 3D rGO/ PPY/CPP20 scaffolds with size of $10 \times 10 \times 1.6 \mathrm{~mm}$ were sterilized under UV radiation for $24 \mathrm{~h}$ and used for MTT assay. Osteoblast-like MC3T3-E1 cells (a clone mouse osteoblastic cell line, ATCC, Rockville, MD, USA) were employed as probe cells to evaluate the biocompatibility and proliferation of the scaffold with or without CPP decoration. The abovementioned scaffolds were placed in a 24-well plate and then the cells were seeded with $10^{6}$ cell per $\mathrm{mL}$ concentration in each well. At a given time (1, 2 and 4 days), $100 \mu \mathrm{L}$ of 3-(4,5-dimethyl-2-thiazolyl)-2,5diphenyl-2- $H$-tetrazolium bromide (MTT, St. Louis, MO, USA) solution was injected into each well. The cells were cultured for another four hours. The lower blue formazan reaction product was dissolved by adding $200 \mu \mathrm{L}$ of dimethyl sulfoxide. Then, the mixed solution was transferred into a 96-well plate, and its absorbance was measured three times using a microplate reader (Bio-Rad iMark) and the average absorbance value was calculated. For comparison, the absorbance of the cells in the culture medium only, was selected as control. The proliferation performance of the as-prepared samples was obtained by comparing the absorbance of the experimental group and control group (set as 100\%).

\subsection{Acridine orange/ethidium bromide (AO/EB) double staining}

The MC3T3-E1 cells were seeded at a density of $1 \times 10^{6}$ cells per $\mathrm{mL}$ in a 24-well plate and allowed to grow on 3D rGO/PPY, 3D $\mathrm{rGO} / \mathrm{PPY} / \mathrm{CPP} 10$ and 3D rGO/PPY/CPP20 scaffolds for 2 and 4 days. All cultured scaffolds were washed three times with PBS and dehydrated with absolute ethyl alcohol before staining with mixed AO/EB solution $\left(100 \mu \mathrm{g} \mathrm{mL}{ }^{-1}\right.$ of $\mathrm{AO}$ and $100 \mu \mathrm{g} \mathrm{mL} \mathrm{m}^{-1}$ of $\mathrm{EB})$ on a microscope slide for $1 \mathrm{~min}$ in the dark, and were observed immediately under fluorescence microscope (OLYMPUS BX53).

\subsection{Preparation of FESEM samples}

After co-culturing for 4 days, the scaffolds (3D rGO/PPY, 3D rGO/ PPY/CPP10, and 3D rGO/PPY/CPP20) were taken out from culture medium and rinsed twice with PBS, immobilized with $2.5 \%$ glutaraldehyde at $4{ }^{\circ} \mathrm{C}$ for $2 \mathrm{~h}$, dehydrated by ethanol of different gradients and finally dried at room temperature. To more clearly observe the cell behaviour of adhesion and proliferation under FESEM, a thin layer of Au film was coated on the scaffold under vacuum to improve the electrical conductivity of the samples.

\subsection{ALP activity assay}

Before the ALP activity assay, 3D rGO/PPY based scaffolds were soaked in PBS solution for three days. Then, the soaking solution was used for alkaline phosphatase expression under a cell imaging system. The activity of ALP was monitored using a BCIP/NBT Alkaline Phosphatase Colour Development Kit (no. C3206, Sigma) on the $7^{\text {th }}$ day. MC3T3-E1 cells were fixed in a formaldehyde fixative solution for $5 \mathrm{~min}$, and then rinsed 3 times with PBS solution. The BCIP/NBT dye solutions were prepared by mixing $10 \mu \mathrm{L}$ of BCIP solution $(300 \times)$ and $20 \mu \mathrm{L}$ of NBT solution $(150 \times)$ in $3 \mathrm{~mL}$ of alkaline phosphatase buffer solution. Next, the fixed cells were placed in BCIP/NBT dye mixture solutions for $30 \mathrm{~min}$ at room temperature in the dark, and then rinsed twice in distilled water. All images were captured on a Nikon digital camera (D90).

\subsection{Statistical analysis}

Statistical analyses were performed using SPSS version 12.0. One-way ANOVA with Duncan's multiple range tests were used to examine differences between groups. The values of $P<0.05$ were considered significant, unless stated otherwise.

\section{Results and discussion}

\subsection{Preparation and characterization of the CPP-modified 3D rGO/PPY scaffold}

Creating a bioactive surface is crucial in bone tissue engineering. In a previous study, we successfully fabricated a 3D 
rGO/PPY scaffold with good osteogenic performance through a simple electrostatic LBL assembly strategy, followed by an electrochemical deposition process using nickel foam as a template. ${ }^{8}$ The prepared 3D rGO/PPY scaffold demonstrated good mechanical properties with hardness of $92.27 \pm 4.03 \mathrm{MPa}$ and Young's modulus of $185.94 \pm 10.76 \mathrm{MPa}^{8}{ }^{8}$ Unfortunately, the $3 \mathrm{D}$ rGO/PPY scaffold does not have enough biological activity. Considering the intriguing calcium binding properties of CPP molecules, in the present study, we assembled the CPP molecules on the developed 3D rGO/PPY scaffold and explored the application of the composite scaffold in bone tissue engineering. The detailed synthesis protocol is illustrated in Fig. 1.

Generally, the developed 3D rGO/PPY scaffold is hydrophobic and electrically neutral. To improve the wettability of the scaffold and introduce the positive or negative functional groups on the scaffold surface, we treated the scaffold with air plasma. After treating with air plasma, some oxygen-containing functional groups (such as $\mathrm{OH}^{-}$and $\mathrm{COO}-$ ) were introduced on the surface of the scaffold, ${ }^{21}$ which enabled us to further tailor the charge state of the 3D rGO/PPY scaffold by adsorbing the positively charged $\mathrm{NH}^{+}$group in PDDA molecules through electrostatic interaction (PDDA is positively charged in water solution, which can be used as a positive ion layer for LBL assembly to attract negatively charged ions and/or molecules ${ }^{19}$ ). After anchoring with PDDA molecules, the surface charged state of $3 \mathrm{D}$ rGO/PPY was reversed from negative to positive. The positively charged surface of the $3 \mathrm{D} \mathrm{rGO} / \mathrm{PPY}^{+}$scaffold allowed it to easily absorb the CPP molecules via the similar electrostatic interactions between the $\mathrm{NH}^{+}$groups in PDDA molecules and the negatively charged groups (amino acid sequence, hydroxyl and phosphate groups) in the CPP bio-molecules at physiological $\mathrm{pH} .{ }^{12}$ Finally, on fixating the CPP molecules on scaffolds with cross-linking agents (including NHS, MES and EDC), ${ }^{13,22}$ the CPP-modified 3D rGO/PYY composite was obtained, and marked as 3D rGO/PPY/CPP.

The chemical state of the 3D rGO/PPY based composite scaffolds were characterized by EDS, FT-IR, and XPS spectroscopy. Fig. 2 shows the elemental mapping of the 3D rGO/PPY/ CPP20 scaffold. Besides those of C, N, and O elements, the signals of S and $\mathrm{P}$ elements can be also notably detected and

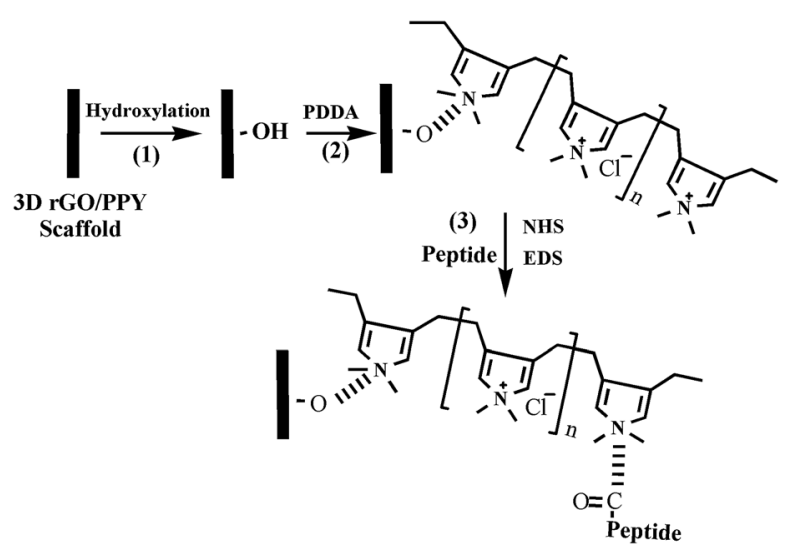

Fig. 1 Schematics to illustrate the procedure to fabricate the 3D rGO/ PPY/CPP scaffold through an electrostatic self-assembly method.

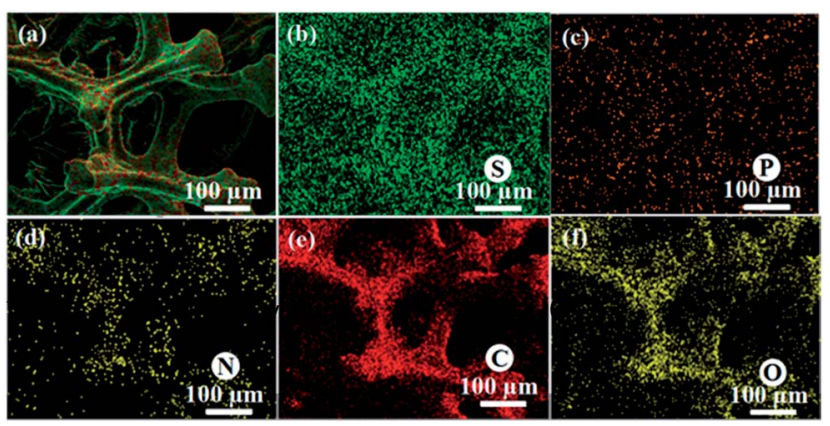

Fig. 2 Elemental mapping of the 3D GO/PPY/CPP 20 composite scaffold: (a) merged image, (b) $\mathrm{S}$, (c) $\mathrm{P}$, (d) $\mathrm{N}$, (e) $\mathrm{C}$, and (f) $\mathrm{O}$ elements.

precisely distributed along the backbone of the 3D rGO/PPY/ CPP20 scaffold (Fig. 2b and c). The S and P elements mainly result from the cross-linking agent of MES and bioactive CPP molecules, suggesting that CPP molecules are successfully anchored to the skeleton surface of the $3 \mathrm{D}$ rGO/PPY matrix.

The existence of the CPP layer can be further supported by FT-IR (Fig. 3). Fig. 3 shows the FT-IR spectra of the 3D rGO/PPY, $\mathrm{CPP}$, 3D rGO/PPY/CPP10, and 3D rGO/PPY/CPP20 scaffolds in the range of $4000-500 \mathrm{~cm}^{-1}$. Fig. 3a shows the FT-IR of 3D rGO/ PPY scaffold. For CPP powder (Fig. 3b), we could see some characteristic peaks at $1290-1100 \mathrm{~cm}^{-1}$, which can be attributed to the $\mathrm{C}-\mathrm{O}$ stretching vibration and the $\mathrm{O}-\mathrm{H}$ deformation vibration. The main two peaks at 1650 and $1455 \mathrm{~cm}^{-1}$ can be ascribed to the $\mathrm{N}-\mathrm{H}$ bending vibration and strong carbonyl $(\mathrm{C}=$ $\mathrm{O})$ /carboxyl C-O stretching vibration, ${ }^{23}$ respectively. For the FTIR spectra of 3D rGO/PPY/CPP10 and 3D rGO/PPY/CPP20 scaffolds, besides the signal resulting from the underlying substrate, the functional groups of CPP molecules can also be detected (Fig. 3c and d), suggesting the successful assembly of CPP molecules on the 3D rGO/PPY scaffold, consistent with the EDS results (Fig. 2).

The chemical composition of the samples was also further investigated by the XPS technique. Fig. 4 shows the XPS data of 3D $\mathrm{rGO} / \mathrm{PPY}, 3 \mathrm{D} \mathrm{rGO} / \mathrm{PPY} / \mathrm{CPP} 10$, and 3D $\mathrm{rGO} / \mathrm{PPY} / \mathrm{CPP} 20$

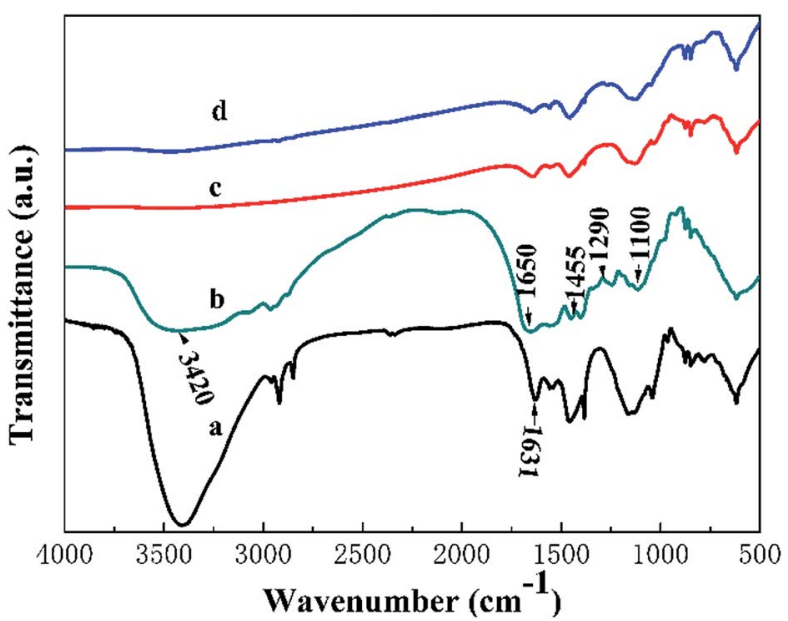

Fig. 3 The FT-IR spectra of 3D rGO/PPY (a), CPP (b), 3D rGO/PPY/ CPP10 (c), and 3D rGO/PPY/CPP20 (d), respectively. 

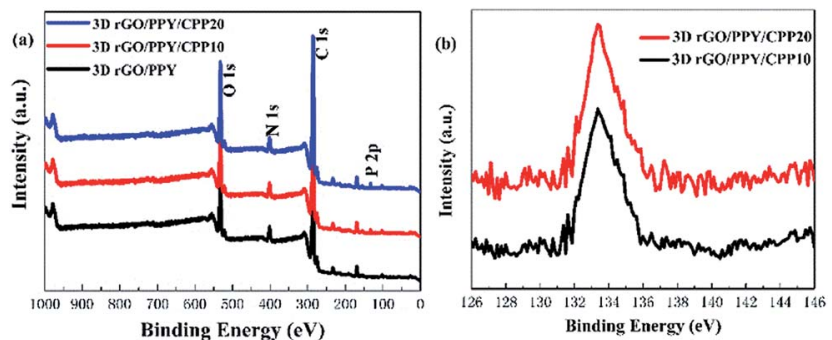

Fig. 4 The full-survey-scan XPS spectrum of 3D rGO/PPY based scaffolds (a), and the P 2p spectra of 3D rGO/PPY/CPP10 and 3D rGO/ PPY/CPP20 (b) composite scaffolds, respectively.

scaffolds. In the full-survey-scan XPS spectrum of the 3D rGO/PPY scaffold (Fig. 4a), except for the signal of C, N, and O elements, no other elements could be detected, while for the survey XPS spectrum of the 3D rGO/PPY/CPP based scaffold, the signal of C, N, O and $\mathrm{P}$ elements were notably observed. The characteristic peak of the $\mathrm{P} 2 \mathrm{p}$ core-level XPS spectrum of 3D rGO/PPY/CPP20 centred at $133.3 \mathrm{eV}$ can be ascribed to the phosphate group in CPP (Fig. 4b). ${ }^{24}$ Also, the characteristic $\mathrm{P} 2 \mathrm{p}$ core-level spectrum can also be obviously observed in the 3D rGO/PPY/CPP10 scaffold, but with relatively lower peak intensity (Fig. 4b). The difference in the peak intensity of the P 2 p core-level spectrum would lead to the huge dissimilarity of the surface physiochemical properties of the two scaffolds, such as water wettability, water uptake and retention rate capability.

\subsection{Wetting properties}

The wetting performance of the scaffold is critically important in bone tissue engineering, which directly determines the performance of cell attachment, spreading and proliferation in vivo/vitro, and osseointegration ability. Water contact angle (WCA) is a major index that reflects the hydrophobicity or hydrophilicity of a matrix. The higher WCA value indicates greater hydrophobicity; otherwise, a greater hydrophilicity. ${ }^{25,26}$ Fig. 5 shows the contact angle values and captured images of the 3D rGO/PPY-based scaffold. The pristine 3D rGO/PPY scaffold demonstrated a high WCA of $109 \pm$ $2^{\circ}$ and was stable for a long time. After decorating with CPP molecules, the WCA values of the scaffold greatly decreased, especially for the 3D rGO/PPY/CPP20 scaffold, which can deliver a relatively low WCA value $85 \pm 3^{\circ}$ under the same measurement conditions. Surprisingly, the WCA of the CPP-anchored composite scaffold quickly reduced to zero after $5 \mathrm{~s}$. These results suggest that after decorating the CPP molecules, the surface properties of the pristine 3D rGO/PPY were changed from hydrophobic to hydrophilic, even super hydrophilic, suggesting the significant role of CPP molecules in tailoring the surface wettability of the scaffold. The reasons for this phenomenon were possibly attributed to the introduction of CPPs on the scaffold which possesses biological active groups such as amino acid sequences, hydroxyl and phosphate groups. ${ }^{27-29}$

\subsection{Water uptake and retention rate}

Fig. 6 shows the water uptake and water retention rate of $3 \mathrm{D}$ rGO/PPY scaffolds with and without CPP decoration. Obviously,

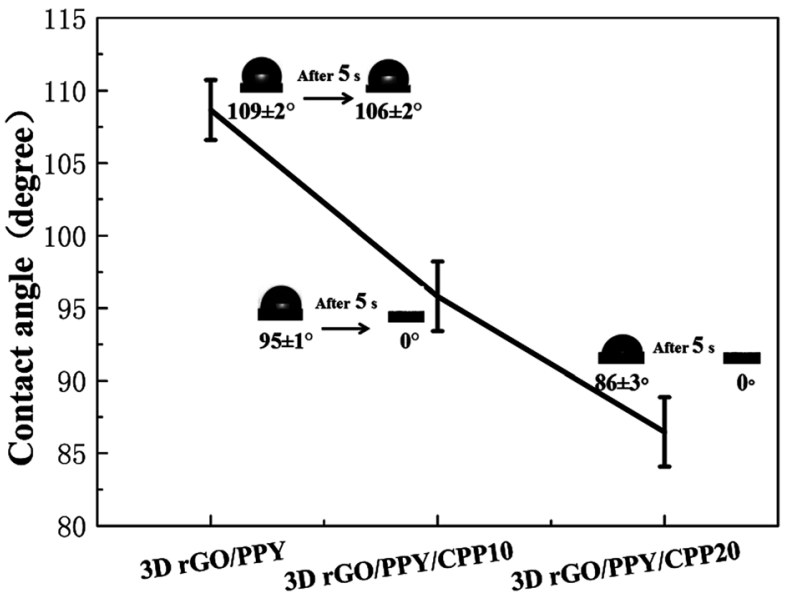

Fig. 5 The difference in contact angles of 3D rGO/PPY based composite scaffolds.

after decoration with different concentrations of CPP molecules, the water uptake rate (the black line in Fig. 6) of 3D rGO/ PPY gradually increased from $4571.7 \pm 231.3 \%$ (3D rGO/PPY) to $6521.3 \pm 530.6 \%(3 \mathrm{D} \mathrm{rGO} / \mathrm{PPY} / \mathrm{CPP} 20)$, suggesting the significant role of CPP molecules in improving the water uptake rate of the scaffolds. A similar trend was observed for the water retention rate. The increase in the water uptake rate of the 3D $\mathrm{rGO} / \mathrm{PPY} / \mathrm{CPP}$ scaffold is closely related to the hydrophilic functional groups in CPP molecules, such as an amino acid sequence, hydroxyl and phosphate groups, which tightly attract and hold the water molecules on the surface of the scaffold..$^{12}$ In literature, the higher water uptake rate of the bio-scaffolds was favourable for cell growth, and good water retention was thought to have a beneficial effect in preventing the accumulation of inflammatory exudates and dehydration. ${ }^{30-32}$

\subsection{Bioactivity evaluation of the CPP-modified 3D rGO/PPY scaffold}

The performance of bone grafting material mainly depends on its osseointegration with surrounding tissues and its biocompatibility with osteoblast cells. ${ }^{33,34}$ To test the osseointegration

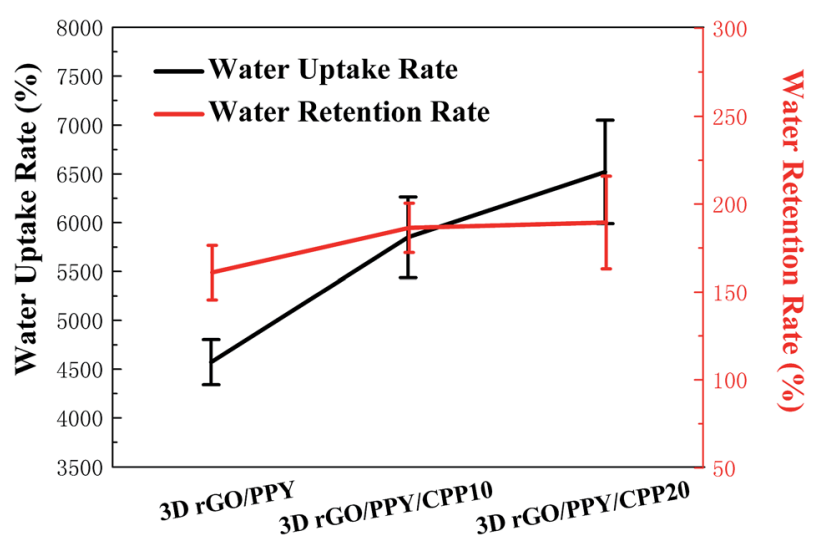

Fig. 6 The water uptake rate and water retention rate of 3D rGO/PPY based composite scaffolds. 
ability and biocompatibility of bone grafting material, researchers usually adopt a simple method to achieve the goal by mineralizing the grafted material in SBF solution and evaluating the formation rate of HA on the scaffold. The faster the HA formation rate, the stronger the osseointegration ability of biomaterials with surrounding tissues. ${ }^{35,36}$ By adopting this method, we also performed the biomimetic mineralization experiments and evaluated the osseointegration ability of the three scaffolds in the present study. Fig. 7 and $\mathrm{S} 1 \uparrow$ show the FESEM images of the 3D rGO/PPY, 3D rGO/PPY/CPP10, and 3D rGO/PPY/CPP20 scaffolds after being soaked in $1.5 \times \mathrm{SBF}$ solution for a period of time. Surprisingly, just at the first day, a compact, highly crystallized nanoparticle layer with size ranging from $100 \mathrm{~nm}$ to $400 \mathrm{~nm}$ was observed on the surface of the 3DrGO/PPY/CPP20 scaffold (Fig. 7i and S1i $\dagger$ ). The XRD pattern indicates that the compact layer consists of HA species with the same composite and identical crystallinity as that of the natural bones in our bodies (Fig. 8c). On the $7^{\text {th }}$ day of mineralization, the crystallinity of the HA layer on the surface of the 3D rGO/PPY/CPP20 scaffold was greatly improved (Fig. 8c). Moreover, the backbone of the scaffold was nearly fully covered with the HA layer with shaggy morphology (Fig. 7l). The highermagnification FESEM image indicates that the spherical HA nanoparticles at the initial mineralization period has fused into large plate-shaped HA blocks on the $7^{\text {th }}$ mineralization day (Fig. S1l†), suggesting the significant role of the 3D rGO/PPY/ CPP20 scaffold in boosting the crystallinity of the HA layer and integrating the HA nanoparticles through healing the defects on the HA crystal surface. For the 3D rGO/PPY/CPP10 scaffold, although some nanoparticles can be observed on the $1^{\text {st }}$ day (Fig. 7e and S1 $\mathrm{e}^{\dagger}$ ), the XRD results confirmed that the composite of the nanoparticle was not HA, but more like octacalcium phosphate (OCP) (Fig. 8b). When we prolonged the mineralization period, the OCP nanoparticles were gradually converted into HA nanoparticles, and on the $5^{\text {th }}$ mineralization day, several characteristic diffraction peaks were detected (Fig. 8b). This implies that the low concentration of CPP molecules on the 3D rGO/PPY scaffold can induce the transformation from OCP to HA crystal. In contrast, only several discrete nanoparticles can be observed on the $1^{\text {st }}$ mineralization day of the 3D rGO/PPY scaffold (Fig. 7a and S1a $\dagger$ ). Moreover, after 7 days of mineralization in $1.5 \times$ SBF solution, there was no characteristic diffractive peak of HA crystal, but only low intensity OCP crystals could be detected (Fig. 8a). Based on these results, it can be concluded that the CPP-decorated 3D rGO/PPY scaffold, especially for the 3D rGO/PPY/CPP20, could effectively promote in vitro mineralization at a fast formation rate. According to above-mentioned results, it can be concluded that at the $1^{\text {st }}$ soaking day, large amounts of HA clusters can be easily detected on the CPP-modified-3D rGO/PPY scaffold (Fig. $7 \mathrm{i}$ and $\mathrm{S} 1 \mathrm{i} \dagger$ ), while on the 3D rGO/PPY scaffold, no obvious HA clusters can be observed, even on the $7^{\text {th }}$ soaking day in SBF solution (Fig. 7d and S1d $\dagger$ ). This indicates that the CPP molecules on the 3D rGO/PPY scaffold have a significant role in accelerating the formation of HA. Based on this fact, a possible formation mechanism for the HA layer formation on the CPPmodified 3D rGO/PPY scaffold was proposed and demonstrated in Fig. S2.† As we described previously, through the
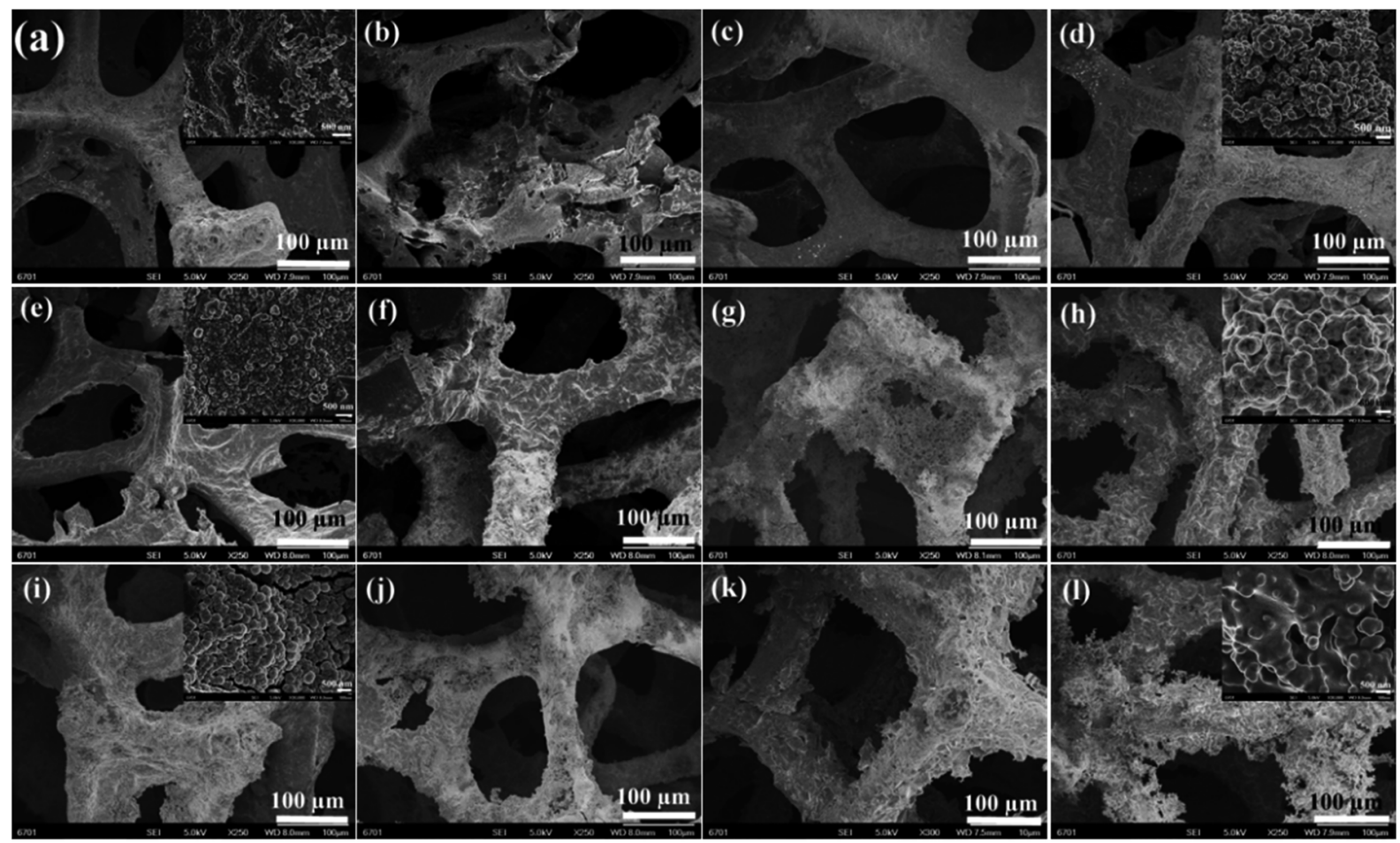

Fig. 7 SEM images of 3D rGO/PPY (the first row), 3D rGO/PPY/CPP10 (the second row), and 3D rGO/PPY/CPP20 (the third row) composite scaffolds after being soaked in $1.5 \times \mathrm{SBF}$ at 37 centigrade for $1,3,5$, and $7 \mathrm{~d}$, respectively. 

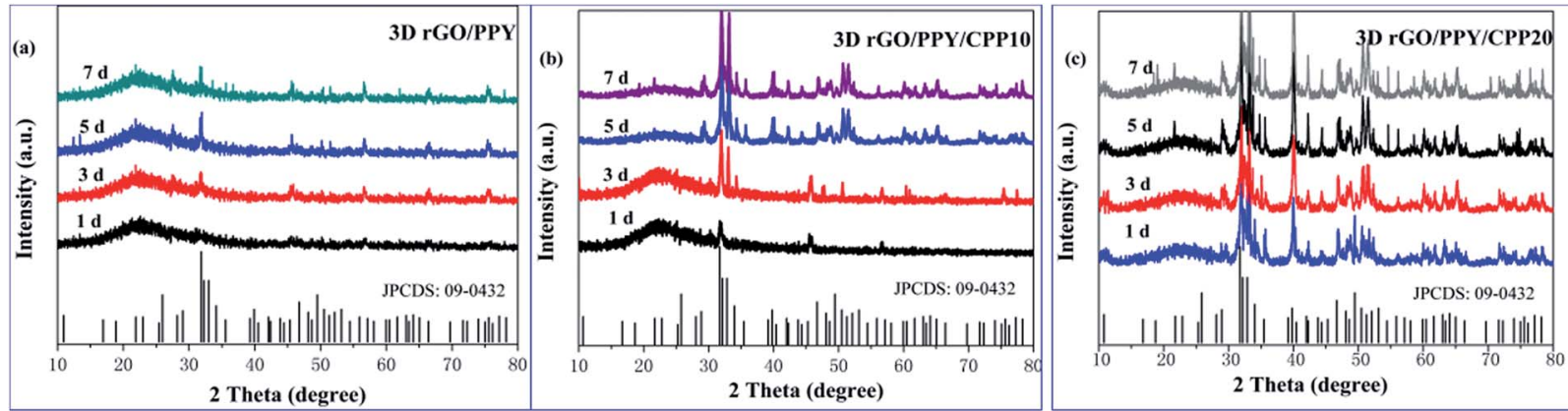

Fig. 8 XRD patterns of 3D rGO/PPY, 3D rGO/PPY/CPP10, and 3D rGO/PPY/CPP20 scaffolds after being mineralized in 1.5 $\times$ SBF for 1, 3, 5, 7 $\mathrm{d}$ and the standard data of HA (JCPDS: 09-0432), respectively.

electrostatic strategy, CPP molecules containing phosphorus groups can be assembled on the $3 \mathrm{D}$ rGO/PPY scaffold. The phosphorus groups have negative charges and can adsorb the positively charged calcium ions in SBF solution through electrostatic integration; new nucleation sites were then created. With auxiliary $\mathrm{OH}^{-}$ions and more $\mathrm{PO}_{4}{ }^{3-}$ and $\mathrm{Ca}^{2+}$ ions absorbed around the nucleation sites, the HA particles were deposited on the CPP-modified 3D rGO/PPY scaffold (Fig. S2 $\dagger$ ). ${ }^{37,38}$

\subsection{Cell biocapability and proliferation ability}

To assess the cell proliferation ability of the 3D rGO/PPY scaffold after decorating with CPP molecules, we conducted the MTT experiment by using the MC3T3-E1 cell as a probe. The proliferation of MC3T3 cells on three prepared scaffolds was measured after being co-cultured for 1, 2, and 4 days. As shown in Fig. 9, at the $1^{\text {st }}$ culture day, the cell number on the CPPdecorated 3D rGO/PPY scaffolds is comparable to that on the pristine 3D rGO/PPY scaffold and control group. Surprisingly, as the culture time was prolonged to 2 days, the cell numbers on 3D rGO/PPY and CPP-decorated 3D rGO/PPY were much higher than that of the control sample, with statistical significance $(P<$

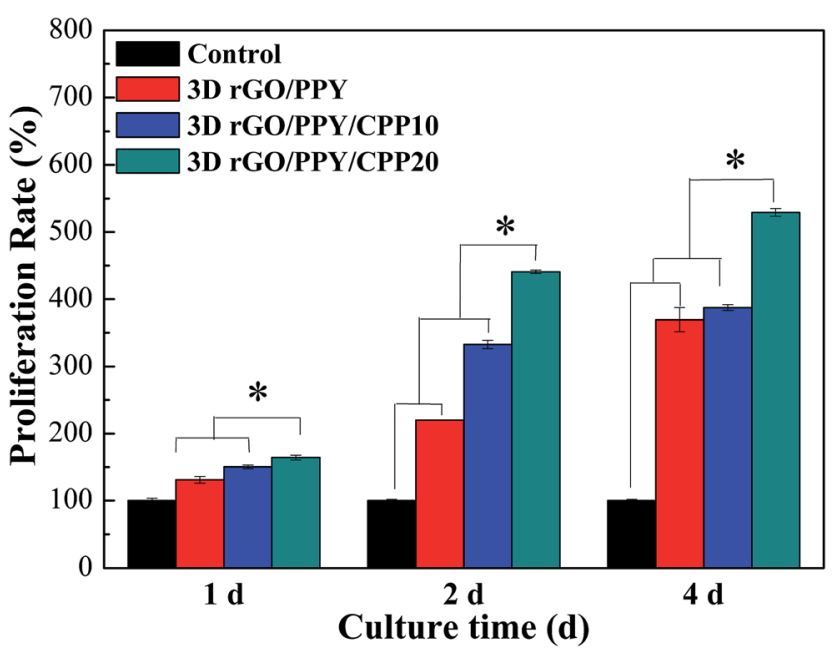

Fig. 9 Cell proliferation assay; the cells were cultured for 1, 2 and 4 days, respectively (asterisk * indicates significance with $P<0.05$, the error bar represents the standard deviation).
0.05). The cell proliferation rate of the $3 \mathrm{D} \mathrm{rGO} / \mathrm{PPY} / \mathrm{CPP} 20$ scaffold is better than that of 3D rGO/PPY/CPP10 scaffold $(P<$ 0.05). On further prolonging the culture period to 4 days, the proliferation rate of the MC3T3-E1 cell on the 3D rGO/PPY/ CPP20 scaffold reached as high as 5.29 times that of the control group, much higher than the 3.88 times of the $3 \mathrm{D} \mathrm{rGO} /$ PPY/CPP10 scaffold and 2.07 times of the 3D rGO/PPY scaffold. These results indicate the good biocompatibility of CPP and its significant role in boosting the bone cell proliferation.

\subsection{AO/EB double staining and fluorescence microscopy}

The cytostatic activity of MC3T3-E1 cells on the abovementioned composite scaffolds was determined by the AO/EB double staining assay. It has been established that acridine orange (AO) is only able to pass through the intact cell membrane of live cells and embed into the nucleus DNA, which gives bright yellow-green fluorescence, while the ethidium bromide (EB) can only penetrate into the membranes of damaged cells and also embed into the nuclear DNA, producing orange-red fluorescence. ${ }^{39,40}$ Fig. 10 shows the fluorescence images of MC3T3-E1 cells cultured at 2 and 4 days on 3D rGO/ PPY, 3D rGO/PPY/CPP10, and 3D rGO/PPY/CPP20 scaffolds, respectively. The fluorescence of MC3T3-E1 cells on all of the scaffolds presents as yellow-green rather than the orange-red color, indicating the non-cytotoxicity of the 3D rGO/PPY-based scaffolds and the good health of MC3T3-E1 cells on all prepared scaffolds. Further observations suggest that there is a large difference in the number of the cells grown on $3 \mathrm{D} \mathrm{rGO} /$ PPY based scaffolds. The cell number on the 3D rGO/PPY based scaffolds on the $4^{\text {th }}$ day (Fig. 10d-f) are much higher than those on the $2^{\text {nd }}$ day (Fig. 10a-c). Also, the cell filopodia are easily observed to extend from the cell bodies on the $4^{\text {th }}$ culture day. According to previous reports, the filopodia at the edge of cell would cause it to tightly bind to the surface of the scaffold, and could greatly improve the adhesion between the cell and scaffold. ${ }^{39}$ In addition, on the $4^{\text {th }}$ day, the cell number on the $3 \mathrm{D}$ $\mathrm{rGO} / \mathrm{PPY} / \mathrm{CPP} 20$ scaffold is also higher than that on the $3 \mathrm{D} \mathrm{rGO} /$ PPY/CPP10 and 3D rGO/PPY scaffold. These findings are consistent with the MTT results, thereby further confirming the non-cytotoxicity of the $3 \mathrm{D}$ rGO/PPY based scaffolds and the 

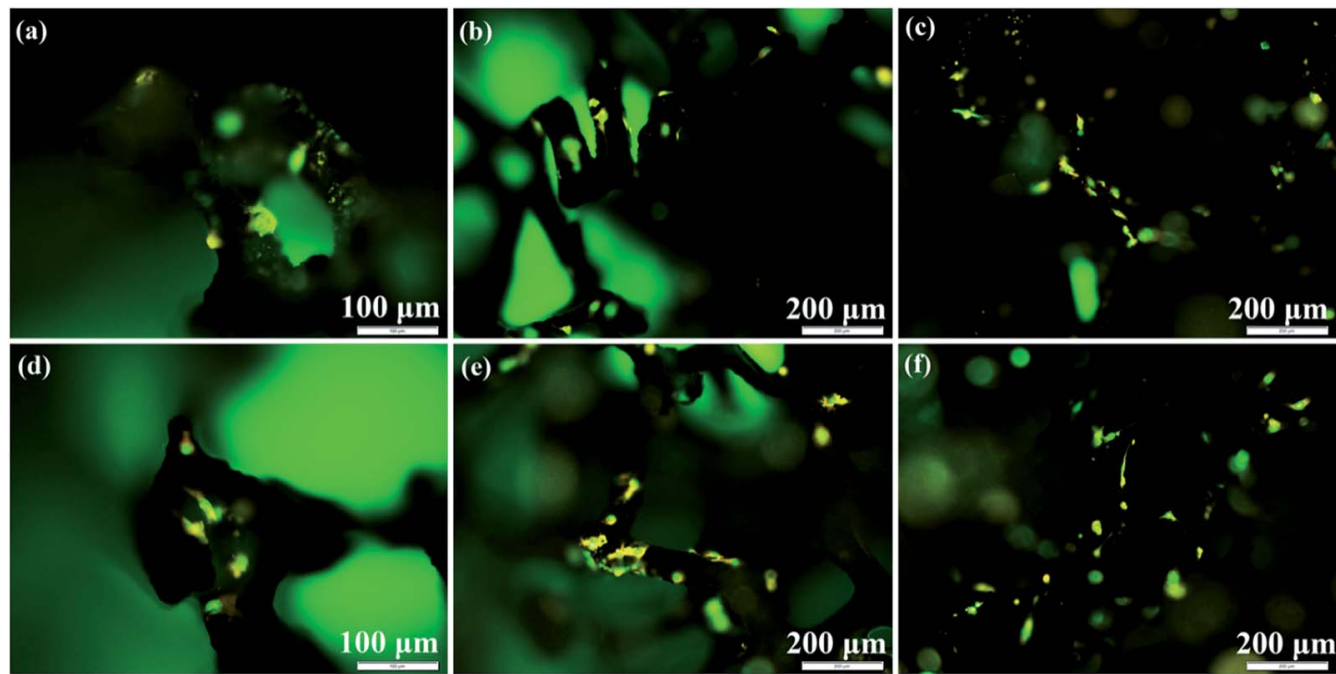

Fig. 10 Fluorescence microscope images of MC3T3-E1 cells after AO/EB double staining on 3D rGO/PPY (a, d), 3D rGO/PPY/CPP10 (b, e), and 3D rGO/PPY/CPP20 (c, f) at 2, and 4 days, respectively.

better cell proliferation ability of the 3D rGO/PPY/CPP20 over the 3D rGO/PPY/CPP10 and 3D rGO/PPY scaffolds.

\subsection{Cell adhesion and proliferation}

The adhesion ability of the MC3T3-E1 cells after being cultured on 3D rGO/PPY, 3D rGO/PPY/CPP10, and 3D rGO/PPY/CPP20 scaffolds were also observed by FESEM. Fig. 11 shows the SEM images of MC3T3-E1 cells cultured on the 3D rGO/PPY based scaffold on day 4. The cells on all prepared scaffold surfaces spread well, but there is a large difference in the pseudopodia length of the cells on different 3D rGO/PPY based scaffolds. On the CPP-modified 3D rGO/PPY scaffold, the pseudopodia can be easily observed (Fig. 11b and c). Especially for the 3D rGO/PPY/CPP20 scaffold, we found that the extremely long pseudopodia covered the whole backbone surface of the scaffold, which is much different from that on the $3 \mathrm{D}$ rGO/PPY
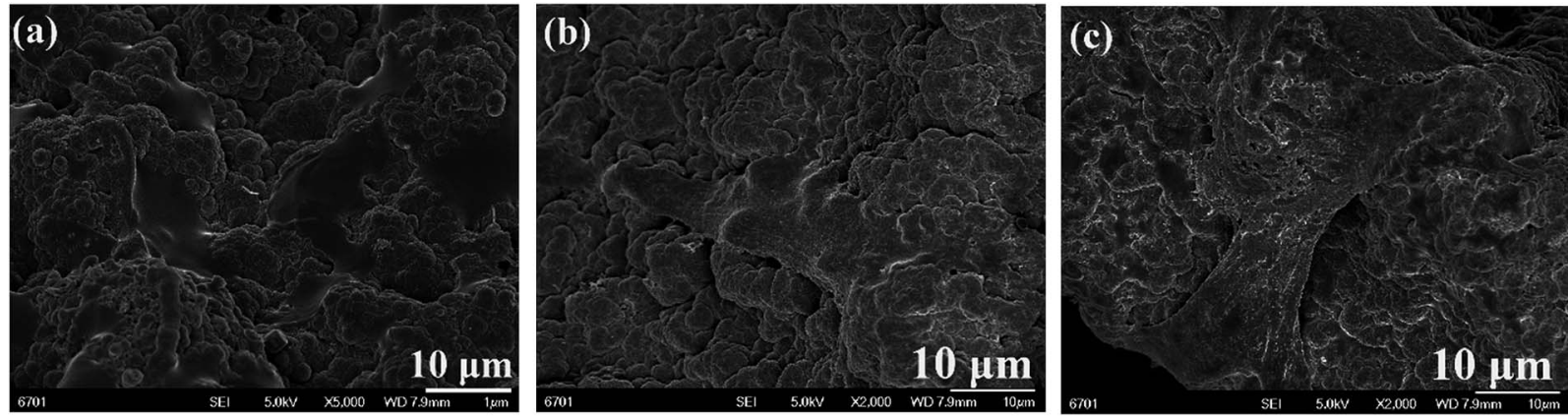

Fig. 11 SEM images of MC3T3-E1 cells cultured on 3D rGO/PPY (a), 3D rGO/PPY/CPP10 (b), and 3D rGO/PPY/CPP20 (c) at 4 days, respectively.
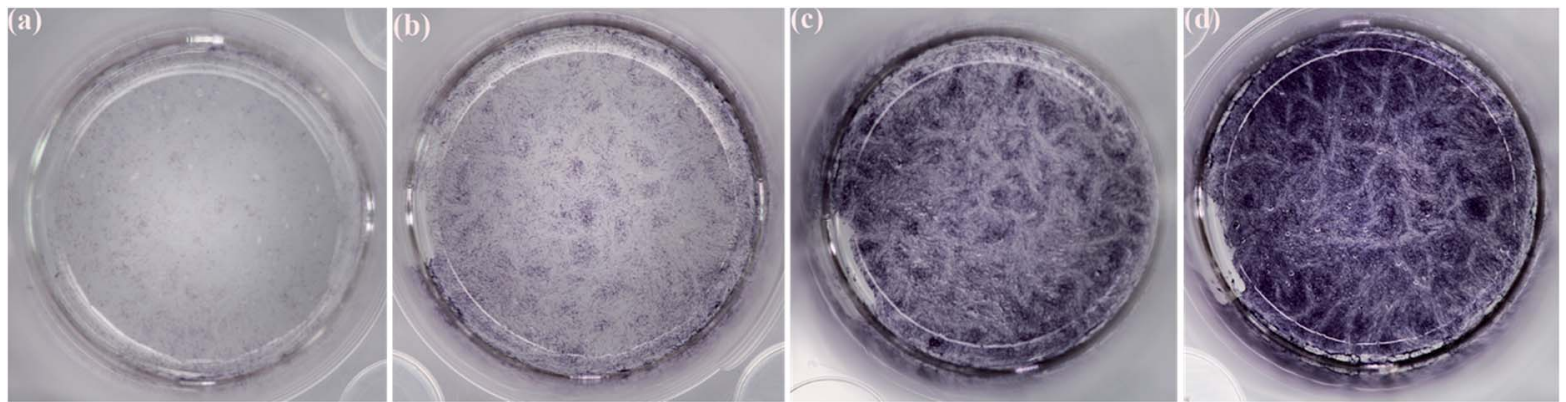

Fig. 12 Digital Images of alkaline phosphatase (ALP) staining of MC3T3-E1 cells treated on the blank control group (a), 3D rGO/PPY group (b), 3D rGO/PPY/CPP10 group (c), and 3D rGO/PPY/CPP group (d) at 7 days post cell seeding, respectively. 
scaffold. This finding suggests that the CPP plays a vital role in enhancing osteoblastic adhesion and proliferation. Also, the CPP-modified 3D rGO/PPY composite scaffold exhibits a better osteoblastic proliferation ability than that on the $3 \mathrm{D} \mathrm{rGO/PPY}$.

\subsection{Osteoblastic differentiation evaluation}

The in vitro osteoblastic differentiation ability of the $3 \mathrm{D} \mathrm{rGO} /$ PPY based scaffolds was evaluated by using the ALP assay, and the result is shown in Fig. 12. ALP activity is an indicator of osteogenesis. The higher ALP activity suggests a more differentiated ability towards mature osteoblasts. ${ }^{41}$ In Fig. 12 , it was found that the number of the dyed MC3T3-E1 cells increased with the increasing CPP concentration on the $7^{\text {th }}$ day of culture. Particularly, the CPP-modified 3D rGO/PPY (Fig. 12c and d) composite scaffold exhibited a higher ALP activity than that of the 3D rGO/PPY (Fig. 12b) and control group (Fig. 12a), possibly due to the presence of CPP on 3D rGO/PPY, which induces the differentiation of MC3T3-E1 cells towards mature osteoblasts. ${ }^{41}$ The results indicate that the CPP modified 3D $\mathrm{rGO} / \mathrm{PPY}$ composite scaffolds can more effectively boost the osteogenic differentiation of pre-osteoblastic MC3T3-E1 cells into mature osteoblasts, compared to the 3D rGO/PPY and control group.

\section{Conclusions}

We have successfully modified the 3D rGO/PPY scaffold through a simple, but low-cost electrostatic self-assembly method using the bioactive CPP molecules. After grafting the CPP molecules, the developed 3D rGO/PPY/CPP scaffold, particularly the 3D rGO/PPY/CPP20 scaffold, demonstrated excellent hydrophilic behaviour and water uptake performance, much better than that of $3 \mathrm{D} \mathrm{rGO/PPY.} \mathrm{For} \mathrm{the} \mathrm{in} \mathrm{vitro} \mathrm{mineralization} \mathrm{assay,} \mathrm{the}$ CPP-modified matrix promoted the rapid formation of hydroxyapatite in $1.5 \times \mathrm{SBF}$ solution; particularly, a compact hydroxyapatite layer was observed on 3D rGO/PPY/CPP20, even on the $1^{\text {st }}$ soaking day. More importantly, the CPP-modified 3D $\mathrm{rGO} / \mathrm{PPY}$ scaffold has excellent ability in boosting the adhesion, proliferation and differentiation of MC3T3-E1 cells, suggesting its promising applications in the bone tissue engineering area.

\section{Acknowledgements}

This work was supported by the Natural Science Foundation of China (81571829), the Medical Subject Fund of Stomatology College of Lanzhou University (201502-3), the open project of State Key Laboratory of Solid Lubrication, Lanzhou Institute of Chemical Physics, Chinese Academy of Sciences (LSL-1505), and the National Natural Science Foundation of China (11472119, 11421062).

\section{References}

1 I. Yip, L. Ma, N. Mattheos, M. Dard and N. P. Lang, Clin. Oral Implants Res., 2015, 26, 606-614.

2 C. Y. Chen, C. J. Ke, K. C. Yen, H. C. Hsieh, J. S. Sun and F. H. Lin, Theranostics, 2015, 5, 643-655.
3 A. Nieto, R. Dua, C. Zhang, B. Boesl, S. Ramaswamy and A. Agarwal, Adv. Funct. Mater., 2015, 25, 3916-3924.

4 N. Shadjou and M. Hasanzadeh, J. Biomed. Mater. Res., Part A, 2016, 104, 1250-1275.

5 G. Tetteh, A. S. Khan, R. M. Delaine-Smith, G. C. Reilly and I. U. Rehman, J. Mech. Behav. Biomed. Mater., 2014, 39, 95110.

6 M.-H. Lee, C. You and K.-H. Kim, Materials, 2015, 8, 11501161.

7 A. B. Kutikov, J. D. Skelly, D. C. Ayers and J. Song, ACS Appl. Mater. Interfaces, 2015, 7, 4890-4901.

8 F. Song, W. Jie, T. Zhang, W. Li, Y. Jiang, L. Wan, et al., RSC Adv., 2016, 6, 92804-92812.

9 G. Khang, J.-H. Choee, J. M. Rhee and H. B. Lee, J. Appl. Polym. Sci., 2002, 85, 1253-1262.

10 I. Wall, N. Donos, K. Carlqvist, F. Jones and P. Brett, Bone, 2009, 45, 17-26.

11 M. Hallman and A. Thor, J. Periodontol., 2008, 47, 172-192.

12 M. A. Flierl, W. R. Smith, C. Mauffrey, K. Irgit, A. E. Wiliams, E. Ross, et al., J. Orthop. Surg. Res., 2013, 8, 33.

13 M. Kantlehner, P. Schaffner, D. Finsinger, J. Meyer, A. Jonczyk, B. Diefenbach, et al., ChemBioChem, 2000, 1, 107-114.

14 Z. Fan, J. Wang, Z. Wang, Z. Li, Y. Qiu, H. Wang, et al., J. Phys. Chem. C, 2013, 117, 10375-10382.

15 Y. Cao, J. Miao, G. Liu, Z. Luo, Z. Xia, F. Liu, et al., J. Agric. Food Chem., 2017, 65, 2307-2314.

16 G. Tulipano, O. Bulgari, S. Chessa, A. Nardone, D. Cocchi and A. Caroli, Regul. Pept., 2010, 160, 168-174.

17 H. Tsuchita, T. Suzuki and T. Kuwata, Br. J. Nutr., 2001, 85, 5-10.

18 K. Ren, J. Ji and J. Shen, Biomaterials, 2006, 27, 1152-1159.

19 B. Hu, S. S. Wang, J. Li, X. X. Zeng and Q. R. Huang, J. Phys. Chem. B, 2011, 115, 7515-7523.

20 D. Depan, P. K. Surya, B. Girase and R. D. Misra, Acta Biomater., 2011, 7, 2163-2175.

21 Y.-C. Lee, H. U. Lee, M. Lee, J. Kim and Y. S. Huh, J. Nanosci. Nanotechnol., 2016, 16, 1392-1395.

22 T. Andersson, F. Agholme, P. Aspenberg and P. Tengvall, J. Mater. Sci.: Mater. Med., 2010, 21, 3029-3037.

23 A. Edelmann and B. Lendl, J. Am. Chem. Soc., 2002, 124, 14741-14747.

24 X. W. Fan, L. J. Lin, J. L. Dalsin and P. B. Messersmith, J. Am. Chem. Soc., 2005, 127, 15843-15847.

25 B. S. Yilbas, M. R. Yousaf, H. Ali and N. Al-Aqeeli, J. Appl. Polym. Sci., 2016, 133, 44015.

26 L. He, C. Mu, J. Shi, Q. Zhang, B. Shi and W. Lin, Int. J. Biol. Macromol., 2011, 48, 354-359.

27 L. Qin, P. Lin, Y. Zhang, G. Dong and Q. Zeng, Appl. Surf. Sci., 2013, 268, 79-86.

28 R. Olivares-Navarrete, S. L. Hyzy, P. J. Slosar, J. M. Schneider, Z. Schwartz and B. D. Boyan, Spine, 2015, 40, 399-404.

29 S. Amjadian, E. Seyedjafari, B. Zeynali and I. Shabani, Int. J. Pharm., 2016, 507, 1-11.

30 D. Depan, P. K. Surya, B. Girase and R. D. Misra, Acta Biomater., 2011, 7, 2163-2175. 
31 R. Jayakumar, M. Prabaharan, P. T. Sudheesh Kumar, S. V. Nair and H. Tamura, Biotechnol. Adv., 2011, 29, 322-337.

32 X. Zeng, Y. Fan, G. Wu, C. Wang and R. Shi, J. Hazard. Mater., 2009, 169, 1022-1028.

33 R. Agarwal and A. J. Garcia, Adv. Drug Delivery Rev., 2015, 94, 53-62.

34 H. Li, J. Fan, L. Sun, X. Liu, P. Cheng and H. Fan, Biomaterials, 2016, 106, 180-192.

35 T. Kokubo and H. Takadama, Biomaterials, 2006, 27, 29072915.

36 P. Roach, D. Eglin, K. Rohde and C. C. Perry, J. Mater. Sci.: Mater. Med., 2007, 18, 1263-1277.
37 D. G. Shchukin, G. B. Sukhorukov and H. Mohwald, Chem. Mater., 2003, 15, 3947-3950.

38 W. Cui, X. Li, C. Xie, H. Zhuang, S. Zhou and J. Weng, Biomaterials, 2010, 31, 4620-4629.

39 N. I. Aminuddin, R. Ahmad, H. M. Ansari, N. M. Zain, S. A. Akbar and B. Pingguan-Murphy, Mater. Des., 2016, 94, 274-279.

40 V. SimiĆ, S. KolareviĆ, I. BrČEski, D. JeremiĆ and B. VukoviĆ-GaČIĆ, Turk. J. Biol., 2016, 40, 661-669.

41 H. M. Kim, D. H. Kim, H. J. Han, C. M. Park, S. R. Ganipisetti, M. Valan Arasu, et al., Molecules, 2016, 22, 42. 